\title{
Symmetric Tensor Algebras and Integral Decompositions
}

\author{
By
}

\author{
Daniel A. Dubin* and Mark A. Hennings**
}

\begin{abstract}
Let $\lambda$ be a perfect sequence space closed under the Cauchy convolution product, with respect to which it is a topological algebra with jointly continuous product. Suppose, in addition, that $\lambda$ is type $(h)$ and $\lambda \subset h$. Let $E[t]$ be a nuclear Fréchet space, with the topology $t$ defined by the seminorms $\left\{p_{r}{ }^{(1)}: r \geq 0\right\}$. The corresponding regular tensor algebra is

$$
T(E, \lambda)=\left\{x \in \Pi_{n \geq 0} \hat{\otimes}^{n} E:\left[p_{r}^{(n)}\left(x_{n}\right)\right] \in \lambda, r \geq 0\right\},
$$

equipped with the topology $t\left(E, \lambda^{x}\right)$ determined by the seminorms

$$
p_{u, r}(x)=\sum_{n \geq 0}\left|u_{n}\right| p_{r}{ }^{(n)}\left(x_{n}\right), \quad(u \in \lambda \times) .
$$

The algebra product is

$$
(x y)_{n}=\sum_{0 \leq k \leq n} x_{n-k} \otimes y_{k} .
$$

If $S_{n}$ is the symmetrizing operator on $\hat{\otimes}^{n} E$ for all $n \geq 0$, we consider the symmetric subalgebra $S[T(E, \lambda)]$, where $S\left(x_{n}\right)=\left(S_{n} x_{n}\right)$.

We show that functionals on $T(E, \lambda)$ and $S[T(E, \lambda)]$ can be represented by complete finite complex Borel measures if they are continuous with respect to a certain locally convex topology $\hat{t}\left(E, \lambda^{\times}\right)$on $T(E, \lambda)$ and its restriction $\hat{t}_{S}\left(E, \lambda^{\times}\right)$to $S\left[T\left(E, \lambda^{\times}\right)\right]$respectively. The topology $\hat{t}\left(E, \lambda^{\times}\right)$is coarser than $t\left(E, \lambda^{\times}\right)$and non-Hausdorff, whereas $\hat{t}_{S}\left(E, \lambda^{\times}\right)$is Hausdorff.

If the algebraic positive cone in $\lambda$ is normal, then so is the positive cone on $S[T(E, \lambda)]$. Using the isomorphism $\lambda \cong T(C, \lambda)$, we show that the cone in $S[T(E, \lambda)]$ is normal for all nuclear Fréchet spaces $E[t]$ if and only if $t\left(\boldsymbol{C}, \lambda^{\times}\right)=\hat{t}\left(\boldsymbol{C}, \lambda^{\times}\right)$.
\end{abstract}

\section{§1. Introduction}

In an earlier work [4] we introduced the class of regular tensor algebras, which are related to the algebras of quantum field theory [3]. Certain questions of interest for the quantum field algebras may therefore usefully be considered for regular tensor algebras.

Communicated by H. Araki, February 16, 1989.

* The Open University, Milton Keynes MK7 6AA, England.

** Sidney Sussex College, Cambridge CB2 3HU, England. 
One such question is to discover which linear functionals may be represented by complex measures. Borchers and Yngvason [3] have shown these to be those functionals which are continuous with respect to the finest locally convex Hausdorff topology on the subalgebra, coarser than the original, under which the algebra product is jointly continuous. This topology turns out to be the finest such that the closed positive cone is normal.

The same question is considered for regular tensor algebras in this paper. A similar answer is found. The topology in question has a slightly different interpretation now, but is closely related to the normality of the closed positive cone.

We have organized this paper as follows. The remainder of this section contains background material from [4]. In $\$ 2$ we consider the positive cone on the sequence space $\lambda$ associated to a regular tensor algebra. The new topology on the tensor algebra is discussed in $\S 3$, along with the decomposition of linear functionals. In $\S 4$ we introduce the symmetric tensor algebra, and in $\S 5$ we consider its order properties and the integral representation theorem for it.

Much of this paper follows the work of Borchers and Yngvason in [3] quite closely. There are some differences worth noting, however. For one thing, we do not have to restrict ourselves to nuclear Fréchet spaces with a basis. For another, characters on a regular tensor algebra are not automatically continuous, as is the case for the quantum field algebras. They are always measurable, though, and so the integrability theories are quite similar. We introduce a constraint on the sequence space sufficient to ensure strong continuity of the characters.

$\mathbb{D e f i m i t i o n ~} \mathbb{1} .1$. Let $\phi, \omega$ be the sequence spaces of all terminating, respectively all, complex sequences. All sequence spaces $\phi \subset \lambda \subset \omega$ that we consider will be assumed to have the following properties, $c f,[4,5]$.

1.耳. $\lambda$ is equipped with its normal topology, denoted $\tau\left(\lambda^{\times}\right)$, which is determined by the seminorms

$$
a \mapsto p_{u}(a)=\sum_{n \geq 0}\left|u_{n} a_{n}\right|, \quad\left(u \in \lambda^{\times}\right) .
$$

By $\lambda^{\times}$we mean the Köthe dual of $\lambda$.

H.1.b. $\lambda$ is to be perfect, which means that $\lambda=\lambda^{\times \times}$.

1.1.c. $\lambda$ is to be type $(h)$, meaning that if $a \in \lambda$, then $\left(2^{n} a_{n}\right) \in \lambda$.

1.1.d. Let $h$ be the sequence space tsuch hat $a \in h$ if and only if the complex function $z \mapsto \sum_{n \geq 0} a_{n} z^{n}$ is entire analytic. Thus a sequence is an element 
of $h$ if and only if $e^{k n} a_{n} \rightarrow 0$ for all $k=0,1,2, \cdots . \quad N B$ : we shall assume that $\lambda \subset h$ in all that follows.

1.1.e. $\lambda$ is to be a Cauchy algebra. This means that for all $a, b \in \lambda$, we have $a * b \in \lambda$, where the product is Cauchy's convolution product

$$
(a * b)_{n}=\sum_{0 \leq k \leq n} a_{n-k} b_{k} .
$$

1.1.f. The product on $\lambda$ is to be jointly continuous. That is, for any $u \in \lambda^{\times}$ there exist $v, w \in \lambda^{\times}$such that for all $m, n \geq 0$ the inequality $\left|u_{m+n}\right| \leq\left|v_{m} w_{n}\right|$ holds.

Definition 1.2.a. By E[t] we shall mean a nuclear Fréchet space with the topology $t$ determined by an increasing sequence of Hilbertian seminorms $\left\{p_{r}^{(1)}\right.$ : $r \geq 0\}$. We assume, further, that $E[t]$ has a continuous involution, indicated as $x \mapsto x^{*}$, and it is no loss of generality to assume that the seminorms are *-symmetric.

1.2.b. The n-fold tensor product of $E$ with itself, completed in the projective tensor product topology, is indicated as $\hat{E}_{n}=\hat{\otimes}^{n} E$. We shall take it that the topology on $\hat{E}_{n}$ is determined by the seminorms $p_{r}^{(n)}=\bigotimes_{\sigma}^{n} p_{r}^{(1)}$, where $\sigma$ indicates the Hilbertian tensor product topology. On occasion we shall employ the injective tensor product of the seminorms, writing $p_{r, \varepsilon}^{(n)}=\bigotimes_{\varepsilon}^{n} p_{r}^{(1)}$, which again determines the same topology on $\hat{E}_{n}$.

For $n=0$ we adopt the conventions $\hat{E}_{0}=\boldsymbol{C}$ and $p_{r}^{(0)}(z)=|z|$ for all $z \in \boldsymbol{C}, r \geq 0$.

The continuous linear extension of $\left(x_{1} \otimes \cdots \otimes x_{n}\right)^{*}=x_{n}^{*} \otimes \cdots \otimes x_{1}^{*}$ determines a continuous involution on $\hat{E}_{n}$, denoted $y_{n} \mapsto y_{n}^{*}$.

1.2.c. The tensor algebra associated with $E$ and $\lambda$ is defined to be the linear space

$$
T(E, \lambda)=\left\{x \in \Pi_{n \geq 0} \hat{\otimes}^{n} E:\left[p_{r}^{(n)}\left(x_{n}\right)\right] \in \lambda, r \geq 0\right\},
$$

equipped with the product

$$
(x y)_{n}=\sum_{0 \leq k \leq n} x_{n-k} \otimes y_{k},
$$

and the continuous involution $\left(x_{n}\right)^{*}=\left(x_{n}^{*}\right)$.

Proposition 1.3.a. $T(E, \lambda)$ has a multiplicative identity

$$
e=(1,0,0, \cdots) \text {, }
$$

no zero divisors, a trivial centre $\mathbb{C} e$, and $0, e$ are its only idempotents.

1.3.b. Let $t\left(E, \lambda^{\times}\right)$be the locally convex Hausdorff topology on $T(E, \lambda)$ determined by the seminorms 


$$
p_{u, r}(x)=\sum_{n \geq 0}\left|u_{n}\right| p_{r}^{(n)}\left(x_{n}\right) .
$$

Equipped with this topology, $T\left(E, \lambda^{\times}\right)$is a nuclear topological $*_{\text {-algebra with }}$ jointly continous product.

When $\lambda$ is Fréchet, so is $T(E, \lambda)$, and hence it is also barrelled, bornological, Mackey, Montel, and reflexive. In such cases its dual, equipped with the strong dual topology, is barrelled, bornological, complete DF, Mackey, Montel, nuclear, and reflexive, $c f,[4]$.

\section{§2. Order Properties of $\lambda$}

The analysis of the order properties of $\lambda$ in [4] was based on the choice of

$$
\lambda_{+}=\left\{a \in \lambda: a_{n} \geq 0, \forall n \geq 0\right\}
$$

as positive cone. Both the positive cone of $T(E, \lambda)$,

$$
K(E, \lambda)=\left\{\sum_{i<\infty} a(i)^{*} a(i): a(i) \in T(E, \lambda)\right\}
$$

and its dual cone

$$
K(E, \lambda)^{\prime}=\{T \in T(E, \lambda): T(x) \geq 0, \forall x \in K(E, \lambda)\},
$$

are constructed quite differently. For this reason, the order properties of $\left[\lambda, \lambda_{+}\right]$and $[T(E, \lambda), K(E, \lambda)]$ are not entirely consonant. Our principal results in [4] were these:

Proposition 2.1.a. Equipped with the normal topology, $\lambda_{+}$is a closed, normal, strict-b cone, and $\lambda_{h}$ is a vector lattice.

2.1.b. $K(E, \lambda)$ is a proper strict-b cone for the usual topology $t\left(E, \lambda^{\times}\right)$. Its closure, $\overline{K(E, \lambda)^{\lambda}}$, is proper if and only if $K(E, \lambda)^{\prime}$ separates the points of $T(E, \lambda)$. $A$ sufficient condition for this is that $\lambda \subset l^{\infty}$. Thus, eg, the closed cone is proper when $\lambda$ is $\phi$ or $h$, but is improper for $\lambda=\omega$.

2.1.c. $K(E, \lambda)$ is normal if and only if the states of $T\left(E, \lambda^{\times}\right)$determine the topology $t\left(E, \lambda^{\times}\right)$. For this it is sufficient that $\lambda$ be of type $N 1, N 2$, or $N 3$.

The order properties of $\lambda$ and $T(E, \lambda)$ will be more naturally congruent if we consider the order relation on $\lambda$ to be given by its algebraic cone, viz.,

$$
K(\lambda)=\left\{\sum_{i<\infty} \overline{a(i)} * a(i): a(i) \in \lambda\right\},
$$

rather than by $\lambda_{+}$. The reason is clear from the next result.

Lemma 2.2. $T(\mathbb{C}, \lambda)\left[t\left(\mathbb{C}, \lambda^{\times}\right)\right]=\lambda\left[\tau\left(\lambda^{\times}\right)\right]$is a tvs isomorphism, under which 
$K(\boldsymbol{C}, \lambda)=K(\lambda)$

In view of this, the results of Proposition 2.1 apply to the pair $[\lambda, K(\lambda)]$, yielding the following.

Corollary 2.3.a. $K(\lambda)$ is a proper strict-b cone with respect to the topology $\tau\left(\lambda^{\times}\right)$. It is generating, and as $\lambda \subset h$, the $\tau\left(\lambda^{\times}\right)$-closure of $K(\lambda)$ is proper.

2.3.b. If $\lambda$ is of type $N 1, N 2$, or $N 3$, then $K(\lambda)$ is normal.

2.3.c. The dual cone, $K(\lambda)^{\prime}=K(C, \lambda)^{\prime}$, consists of the collection of those sequences in $\lambda^{\times}$which are of positive type, viz, $u \in K(\lambda)^{\prime}$ if and only if for all $a \in \phi$,

$$
\sum_{i, j \geq 0} u_{i+j} \bar{a}_{i} a_{j} \geq 0 \text {. }
$$

We shall prove that functionals on the symmetric subalgebra of $T(E, \lambda)$ possess an integral decomposition with respect to a complex measure on $E_{h}^{\prime}$ provided that they are continuous with respect to a certain topology $\hat{t}\left(E, \lambda^{\times}\right)$. Because of Lemma 2.2, we can define this topology for $\lambda$ itself, writing $\hat{t}\left(\boldsymbol{C}, \lambda^{\times}\right)$ $=\hat{\tau}\left(\lambda^{\times}\right)$in this case. As we shall now show, $\tau\left(\lambda^{\times}\right)=\hat{\tau}\left(\lambda^{\times}\right)$if and only if $K(\lambda)$ is normal. The existence of integral representations for abelian functionals on $T(E, \lambda)$ is a result of this important relation between order and continuity on $\lambda$. This connexion was first discovered by Borchers and Yngvason in their study of the Euclidean quantum field algebra [3]. For that tensor algebra, only the sequence spaces $\phi$ and $\omega$ occur, which tends to obscure the fact that the ordercontinuity properties of the tensor algebra reflect those of $\lambda$.

Definition 2.4.a. $B y \Lambda^{\times}$we shall mean the strictly positive sequences in $\lambda^{\times}$:

$$
\Lambda^{\times}=\left\{u \in \lambda^{\times}: u_{n}>0, \forall n \geq 0\right\} .
$$

Each such sequence $u$ determines an extended real-valued function $F_{u}: \boldsymbol{R} \mapsto$ $[0, \infty]$ given by

$$
F_{u}(t)=\sum_{n \geq 0}\left(u_{n}\right)^{-1}|t|^{n} .
$$

2.4.b. We denote the standard isomorphism $\phi \cong C[X]$ by $a \mapsto P_{a}(X)$, ie,

$$
P_{a}(X)=\sum_{n \geq 0} a_{n} X^{n},
$$

where $X$ is an indeterm nate. By the usual abuse of notation, we identify the polynomial $P_{a}(X)$ with the polynomial function $t \mapsto P_{a}(t)$ for $t \in R$, and in the next section with the algebra element $P_{a}(x) \in T(E, \lambda)$, all $x \in T(E, \lambda)$.

2.4.c. For any $u \in \Lambda^{\times}$we consider the extended real-valued function on $\lambda$ given by 


$$
\|a\|_{u}=\sup \left\{\left|P_{a}(t)\right| / F_{u}(t): t \in \mathbb{R}\right\}
$$

for $a \in \lambda$.

We prove that the $\|\cdot\|_{u}$ are all seminorms.

Lemma 2.5. The $\left\{\|\cdot\|_{u}: u \in \Lambda^{\times}\right\}$constitute a family of seminorms on $\lambda$. If we write $\hat{\tau}\left(\lambda^{\times}\right)$for the locally convex Hausdorff topology they determine, then $\hat{\tau}\left(\lambda^{\times}\right) \subset \tau\left(\lambda^{\times}\right)$.

Proof. The original topology $\tau\left(\lambda^{\times}\right)$is determined by the seminorms $p_{u}(a)$ $=\sum_{n \geq 0}\left|u_{n} a_{n}\right|$, for all $u \in \lambda^{\times}$. Restrict them to $\phi$, using the same symbol. Let $a \in \phi$, and we may suppose that $a_{n}=0$ for all $n>N$. Then

$$
\left|P_{a}(t)\right| \leq p_{n}(a) F_{u}(i), \quad \forall u \in \lambda^{\times}, t \in \mathbb{R} .
$$

If we understand $P_{a}(t) / F_{u}(t)=0$ whenever $F_{u}(t)=\infty$, then $\|a\|_{u} \leq p_{u}(a)$. We may extend the $\|\cdot\|_{u}$ to $\lambda$, therefore, preserving the seminorm properties and this bound. That $\hat{\tau}\left(\lambda^{\times}\right)$is Hausdorff is elementary.

Theorem 2.6. $K(\lambda)$ is $\tau\left(\lambda^{\times}\right)$-normal if and only if $\hat{\tau}\left(\lambda^{\times}\right)=\tau\left(\lambda^{\times}\right)$.

Proof. A computation shows that if

$$
a=\sum_{i<\infty} \overline{a(i)} * a(i)
$$

is an element of $K(\phi)$, then

$$
P_{a}(t)=\sum_{i<\infty}\left|P_{a(i)}(t)\right|^{2} .
$$

Then if $a, b$, and $b$ - $a$ belong to $K(\phi)$, it follows that $P_{a}(t), P_{b}(t)$, and $P_{b-a}(t)$ are non-negative for all $t \in \mathbb{R}$. Consequently, $\|a\|_{u} \leq\|b\|_{u}$ for all $u \in \Lambda^{\times}$, so $K(\phi)$ is $\hat{\tau}\left(\lambda^{\times}\right)$-normal.

Suppose now that $\tau\left(\lambda^{x}\right)=\hat{\tau}\left(\lambda^{x}\right)$. The closure of $K(\phi)$ in this topology must be normal. As this closure coincides with the $\tau\left(\lambda^{\times}\right)$-closure of $K(\lambda)$ (see [4]), this closure and a forteriori $K(\lambda)$ itself must be $\tau\left(\lambda^{\times}\right)$-normal.

The opposite implication may be inferred from Lemma 3.1 of [3], with the sequences appearing there suitably restricted to $\lambda^{\times}$and $\Lambda^{\times}$.

\section{§3. Integral Decompositions for $\mathbb{T}(\mathbb{E}, \lambda)^{\prime}$}

As in [3], we shall construct a class of functions integrable with respect to the measures which occur in the integral decomposition theory. To start with, every hermitian functional $\psi \in E_{h}^{\prime}$ on $E$ defines a character, which we denote by $M_{\psi}$, on $T(E, \lambda)$ through the formula 


$$
M_{\psi}=\left(\otimes^{n} \psi: n \geq 0\right) .
$$

These characters enable us to associate a nonlinear function $m_{x}: E_{h}^{\prime} \rightarrow \boldsymbol{C}$ to each $x \in T(E, \lambda)$ by setting

$$
m_{x}(\psi)=M_{\psi}(x)
$$

Definition 3.1. By $\boldsymbol{F}$ we shall mean the set of all functions $f: E_{k}^{\prime} \rightarrow C$ of the form

$$
f(\psi)=G\left[m_{x(1)}(\psi), \cdots, m_{x(n)}(\psi)\right]
$$

for all $x(1), \cdots, x(n) \in T(E, \lambda)$, all polynomially bounded continuous functions $G: \boldsymbol{C}^{n} \rightarrow \boldsymbol{C}$, and all $n \geq 1$. With pointwise operations, $\boldsymbol{F}$ is a commutative *algebra.

Given any $f \in \boldsymbol{F}$ as in (3.2), we can always find a polynomial $Q \in \boldsymbol{C}\left[X_{1}\right.$, $\cdots, X_{n}$ ] dominating $G$ so that we have the bound

$$
|f(\psi)| \leq \mid M_{\psi}[Q(x(1), \cdots, x(n)] \mid .
$$

Our aim is to define a topology $\hat{t}\left(E, \lambda^{\times}\right)$for $T(E, \lambda)$ analogous to $\hat{t}\left(\lambda^{\times}\right)$ on $\lambda$. This requires some preliminary definitions.

Definition 3.2.a. For any $u \in \Lambda^{\times}$and $r \geq 0$, define the function $F_{u, r}: E_{h}^{\prime} \rightarrow$ $[0, \infty]$ by

$$
F_{u, r}(\psi)=\left(p_{u, r, \varepsilon}\right)^{\circ}\left(M_{\psi}\right)
$$

By $p_{u, r, q}$ we mean the $T(E, \lambda)$ seminorm

$$
p_{u, r, \varepsilon}(x)=\sum_{n \geq 0}\left|u_{n}\right| p_{r, \varepsilon}^{(n)}\left(x_{n}\right)
$$

based on the injective tensor product topology. Then

$$
\left(p_{u, r, \mathrm{\varepsilon}}\right)^{\circ}(T)=\sup \left\{|T(x)|: p_{u, r, \varepsilon}(x) \leq 1\right\}
$$

defines its polar seminorm.

3.2.b. For any $u \in \Lambda^{\times}$and $r \geq 0$ let $\pi_{u, r}: \boldsymbol{F} \rightarrow[0, \infty]$ be the extended realvalued function given by

$$
\pi_{u, r}(f)=\sup \left\{|f(\psi)| / F_{u, r}(\psi): \psi \in E_{h}^{\prime}\right\},
$$

with the usual convention when $F_{u, r}(\psi)=+\infty$.

These functions define extended real-valued functions $\hat{\pi}_{u, r}: T(E, \lambda) \rightarrow[0, \infty]$ by setting 


$$
\hat{\pi}_{u, r}=\pi_{u, r} \circ m
$$

where $m: T(E, \lambda) \rightarrow F$ is given by $m(x)=m_{x}$.

We have allowed for the possibility that $\hat{\pi}_{u, r}$ is not well-defined everywhere, but this does not in fact occur.

Proposition 3.3. The collection $\left\{\pi_{u, r}: u \in \Lambda^{\times}, r \geq 0\right\}$ constitutes a family of seminorms on $\mathbb{F}$ defining a locally convex Hausdorff topology, written $\hat{t}\left(\boldsymbol{F}, \lambda^{\times}\right)$, with respect to which $\mathbb{F}$ is a topological lattice.

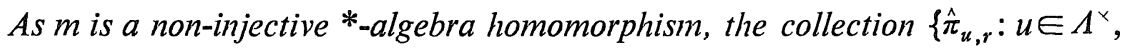
$r \geq 0\}$ consists of seminorms on $T(E, \lambda)$ defining a locally convex non-Hausdorff topology denoted $\hat{t}\left(E, \lambda^{\times}\right)$. It is coarser than the original topology:

$$
\hat{t}\left(E, \lambda^{\times}\right) \subset t\left(E, \lambda^{\times}\right) .
$$

Turning to integral representations, we apply the nuclear spectral theorem to $T(E, \lambda)$ as in [3]. The proofs are those of [3], mutatis mutandis, and so we omit them. Note that $\hat{\pi}_{u, r}$ and $p_{u, r, \varepsilon}$ must be employed.

Proposition 3.4.a. Let $R$ be $a$ positive and $\hat{\tau}\left(\boldsymbol{F}, \lambda^{\times}\right)$-continuous linear functional on $\boldsymbol{F}$. As the Cauchy-Schwarz inequality holds for $R$ we can reconstruct its strongly cyclic GNS representation $\left[\Omega_{R}, \mathscr{D}_{R}, \pi_{R}\right]$.

Let $\boldsymbol{B}$ be the subspace of bounded functions in $\boldsymbol{F}$. Then $\pi_{R}(\boldsymbol{B})^{\prime \prime}$ is a maximal abelian $W^{*}$-algebra on $\mathcal{H}_{R}$, the Hilbert completion of $\mathscr{D}_{R}$.

3.4.b. Write $\Gamma_{R}$ for the spectrum of $\pi_{R}(B)^{\prime \prime}, A \mapsto \hat{A}$ for the Gelfand transform $\pi_{R}(\boldsymbol{B})^{\prime \prime} \rightarrow \mathcal{C}\left(\Gamma_{R}\right)$, and $F \mapsto \stackrel{F}{F}$ for its inverse $\mathcal{C}\left(\Gamma_{R}\right) \rightarrow \pi_{R}(\boldsymbol{B})^{\prime \prime}$. Then there exists a complete, positive, and finite Borel measure $\mu_{R}$ on $\Gamma_{R}$ such that for all $F \in \mathcal{C}\left(\Gamma_{R}\right)$ the equality

$$
\int F d \mu_{R}=\left(\Omega_{R}, \stackrel{v}{F} \Omega_{R}\right)
$$

holds.

3.4.c. The map $V_{R}: \mathcal{C}\left(\Gamma_{R}\right) \rightarrow \pi_{R}(B)^{\prime \prime} \Omega_{R}$ given by'

$$
V_{R}(F)=\stackrel{\check{F}}{\Omega_{R}}
$$

extends to a unitary isomorphism $L^{2}\left(\Gamma_{R}, d \mu_{R}\right) \rightarrow \mathscr{H}_{R}$.

$F$ may be identified with an abelian algebra in $L^{2}\left(\Gamma_{R}, d \mu_{R}\right)$ by defining $\sigma_{R}: F \rightarrow L^{2}\left(\Gamma_{R}, d \mu_{R}\right)$,

$$
\sigma_{R}(f)=V_{R}^{-1}\left[\pi_{R}(f) \Omega_{R}\right]
$$


Using $\sigma_{R}$ and $m: T(E, \lambda) \rightarrow \boldsymbol{F}$ defined above, we obtain the following relation between $T(E, \lambda)$ and $L^{2}\left(\Gamma_{R}, d \mu_{R}\right)$.

Corollary 3.5. The map $\zeta_{R}: T(E, \lambda) \rightarrow L^{2}\left(\Gamma_{R}, d \mu_{R}\right)$ given by $\zeta_{R}=\sigma_{R} \circ m$ is continuous, linear, and multiplicative.

The nuclearity of $t\left(E, \lambda^{\times}\right)$is now invoked to prove the following.

Proposition 3.6.a. There exists a $\mu_{R^{-}}$null set $N$ and an element $K_{x} \in$ $L^{2}\left(\Gamma_{R}, d \mu_{R}\right)$ for each $x \in T(E, \lambda)$, such that $x \mapsto K_{x}(\gamma)$ is a $t\left(E, \lambda^{\times}\right)$-continuous character on $T(E, \lambda)$ for each $r \in \Gamma_{R} \backslash N$.

3.6.b For each $r \in \Gamma_{R} \backslash N$ there exists a unique hermitian functional $\Phi(r) \in$ $E_{h}^{\prime}$ such for all $x \in T(E, \lambda)$ the equality $K_{x}(\gamma)=M_{\Phi(\gamma)}(x)$ holds. We extend $\Phi$ to $\Gamma_{R}$ by defining $\Phi$ to be zero on $N$. This extension is uritten $\Phi: \Gamma_{R} \mapsto E_{h}^{\prime}$.

Using $\Phi$ we transport $\mu_{R}$ from $\Gamma_{R}$ to $E_{h}^{\prime}$, obtaining a positive measure $\nu_{R}$ on $E_{h}^{\prime}$ with the properties

(i) $W \subset E_{h}^{\prime}$ is $\nu_{R^{-}}$-measurable if and only if $\Phi^{-1}(W)$ is $\mu_{R^{-}}$measurable, and so the definition

$$
\nu_{R}=\mu_{R} \circ \Phi^{-1}
$$

is consistent.

(ii) $f \in L^{1}\left(E_{h}^{\prime}, d \nu_{R}\right)$ if and only if $f \circ \Phi \in L^{1}\left(\Gamma_{R}, d \mu_{R}\right)$, and the integrals are related by

$$
\int_{E_{h}^{\prime}} f d \nu_{R}=\int_{\Gamma_{R}} f \circ \Phi d \mu_{R}
$$

(iii) The measure $\nu_{R}$ is finite and complete.

We are now able to state the principal measure theoretic result for the tensor algebra. Part (d) below is the integral decomposition theorem referred to in the introduction.

Proposition 3.7.a. For all $y \in E$, the map $\psi \mapsto \Phi(\psi)(y)$ is $\mu_{R^{-}}$-measurable.

3.7.b. For all $y \in E$, the function $E_{h}^{\prime} \rightarrow \boldsymbol{C} ; \psi \mapsto \psi(y)$ is $\nu_{R}$-measurable.

3.7.c. Every weakly' closed subset of $E_{h}^{\prime}$ is $\nu_{R}$-measurable, and all weakly continuous functions $E_{h}^{\prime} \rightarrow C$ are $\nu_{R^{-}}$measurable.

3.7.d. If $R$ is a $\hat{\boldsymbol{t}}\left(E, \lambda^{\times}\right)$-continuous linear functional on $T(E, \lambda)$, theree xists a complete finite complex Borel measure $\nu_{R}$ on $E_{h}^{\prime}$ such that

(i) $m_{x} \in L^{1}\left(E_{h}^{\prime}, d \nu_{R}\right)$ for all $x \in T(E, \lambda)$;

(ii) all weakly continuous functions $E_{h}^{\prime} \mapsto C$ are $\nu_{R}$-measurable;

(iii) for all $x \in T(E, \lambda)$, 


$$
R(x)=\int_{E_{h}^{\prime}} m_{x} d \nu_{R}
$$

The work of Borchers and Yngvason concerned the quantum field algebra $\sum_{n \geq 0} S\left(R^{d n}\right)$, as noted above [3]. In this case, they showed that the nuclearity implied that the $m_{x}$ were strongly continuous and so their measurability follows from continuity. We now know the $m_{x}$ to be measurable, and we consider next the question of continuity.

Proposition 3.8. The functions $m_{x}: E_{h}^{\prime} \rightarrow C$ are strongly continuous for all $x \in T(E, \phi)$.

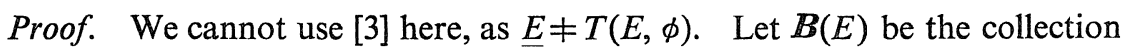
of all bounded subsets of $E$. From the $t v s$ isomorphism $\left(\hat{E}_{n}\right)^{\prime} \cong\left(\otimes_{\pi}^{n} E^{\prime}\right)^{\wedge}$ it follows that $\left\{\bigotimes_{\pi}^{n} p_{B}: B \in \mathbb{B}(E)\right\}$ constitutes a basis of seminorms for the strong topology $\beta\left(\hat{E}_{n}^{\prime}, \hat{E}_{n}\right)$ on $\hat{E}_{n}^{\prime}$. We have introducted the standard notation $p_{B}(\psi)=$ $\sup _{B}\{|\psi(x)|\}$.

Now if $\left(\psi_{a}\right)$ is a net in $E^{\prime}$ which converges in the $\beta\left(E^{\prime}, E\right)$ topology to $\psi$, then $\left(\otimes^{n} \psi_{a}\right)$ is a net in $E_{n}^{\prime}$ similarly converging to $\otimes^{n} \psi_{\text {. }}$ To see this, suppose $\psi, \xi \in E^{\prime}$ and $B \in \mathbb{B}(E)$ are such that $p_{B}(\xi-\psi)<1$. Then

$$
\left(\otimes_{\pi}^{n} p_{B}\right)\left(\otimes^{n} \xi-\otimes^{n} \psi\right) \leq n\left[1+p_{B}(\xi)\right]^{n-1} p_{B}(\xi-\psi),
$$

and convergence follows.

For all $x \in T(E, \phi)$, convergence of $\left(\otimes^{n} \psi_{a}\right)$ implies that

$$
\sum_{n \geq 0}\left(\otimes^{n} \psi_{a}\right)\left(x_{n}\right) \rightarrow \sum_{n \geq 0}\left(\otimes^{n} \psi\right)\left(x_{n}\right)
$$

which is the assertion of the proposition.

Recall that as $E[t]$ is a nuclear Fréchet space, its strong dual may be represented as an inductive limit of Banach spaces

$$
E^{\prime}\left[\beta\left(E^{\prime}, E\right)\right]=\lim \text { ind }\left\{F_{r}\left[q_{r}\right]: r \geq 0\right\},
$$

where $q_{r}$ is the polar norm corresponding to $p_{r}^{(1)}$ on $E$, and $F_{r}$ is the $q_{r}$-completion of $\left\{\psi \in E^{\prime}: q_{r}(\psi)<\infty\right\}$. Using this result we consider $m_{x}$ acting on the $F_{r}$, and then piece together the results.

Lemma 3.9. The function $m_{x}: F_{r}\left[q_{r}\right] \rightarrow C$, obtained by restriction, is continuous for every $r \geq 0$ and all $x \in T(E, \lambda)$.

Proof. Let $\psi, \xi \in F_{r}$ be such that $q_{r}(\xi-\psi)<1$. The bound

$$
\left|\left(\otimes^{n} \xi\right)\left(x_{n}\right)-\left(\otimes^{n} \psi\right)\left(x_{n}\right)\right| \leq n\left[1+q_{r}(\xi)\right]^{n-1} p_{r}^{(n)}\left(x_{n}\right) q_{r}(\xi-\psi)
$$


leads to

$$
\left|m_{x}(\xi)-m_{x}(\psi)\right| \leq\left(\sum_{n \geq 1} n\left[1+q_{r}(\xi)\right]^{n-1} p_{r}^{(n)}\left(x_{n}\right)\right) q_{r}(\xi-\psi),
$$

showing the required continuity.

Corollary 3.10 For all $x \in T(E, \lambda)$, the function $m_{x}: E_{h}^{\prime} \rightarrow C$ is strongly continuous at the origin.

Proof. For every zero neighbourhood $U \subset C$, the set $m_{x}^{-1}(U) \cap F_{r}$ is a zero neighbourhood in $F_{r}$ for all $x, r \geq 0$. Then $m_{x}^{-1}(U)$ is a zero neighbourhood for $\beta\left(E^{\prime}, E\right)$, as was to be shown.

We have not been able to prove the continuity of $m_{x}$ everywhere without further assumptions. The general question remains open. Recall that the measurability of $m_{x}$ has been shown independently, $c f$ Proposition (3.7.d).

A condition that ensures the continuity of $m_{x}$ everywhere is that $\lambda^{\times}$is "nearly" a Cauchy algebra.

Definition 3.11. We shall say that the sequence space $\lambda$ is of type $(M)$ if $u * \delta \in \lambda^{\times}$whenever $u \in \lambda^{\times}$.

Recall that $\delta=(1,1,1, \cdots)$. Of course if $\lambda^{\times}$is a Cauchy algebra it is type $(M)$. An immediate consequence of this definition is the following dominance condition.

Lemma 3.12. If and onlyi $f \lambda$ is of type $(M)$, for every $u \in \lambda^{\times}$there exists $v \in \lambda^{\times}$such that $n \mapsto\left|v_{n}\right|$ is a monotonically increasing positive sequence, and $\left|u_{n}\right| \leq\left|v_{n}\right|$ for all $n \geq 0$.

Proof. If $\lambda$ is of type $(M)$, choose $v=|u| * \delta$, where $(|u|)_{n}=\left|u_{n}\right|$. Conversely, suppose a sequence $v$ as above exists for each $n$. Then $\left|(u * \delta)_{n}\right| \leq$ $(1+n)\left|v_{n}\right|$, proving that $u * \delta \in \lambda$.

The following lemma is the crux of the argument.

Lemma 3.13. If $\lambda$ is of type $(M)$, then given any $x \in T(E, \lambda)$ and $\psi \in E^{\prime}$, we can find an $x(\psi) \in T(E, \lambda)$ such that

$$
m_{x}(\psi+\xi)-m_{x}(\psi)=\left[m_{x}(\xi)-m_{x}(0)\right]+\left[m_{x(\psi)}(\xi)-m_{x(\psi)}(0)\right]
$$

for all $\xi \in E^{\prime}$.

Proof. From the definition of the $\varepsilon$-seminorms it follows that, given any $r \geq 0$ and $n \geq 1$ we can find a $t \geq 0$ such that $\psi \in F_{t}\left[q_{t}\right]$ and 


$$
\begin{gathered}
p_{s, \mathrm{\varepsilon}}^{(r)}\left[\left(\begin{array}{c}
r+n \\
r
\end{array}\right)\left[I_{r} \otimes\left(\otimes^{n} \psi\right)\right]\left(x_{r+n}\right)\right] \\
\leq\left(\begin{array}{c}
r+n \\
r
\end{array}\right) q_{t}\left(\psi^{n}\right)^{n} p_{t+s, \mathrm{e}}^{(r+n)}\left(x_{r+n}\right)
\end{gathered}
$$

for all $s \geq 0, x_{r+n} \in \hat{E}_{r+n}$.

Observing that if we sum over all $n$ convergence obtains, we may define $x(\psi)$ by

$$
x(\psi)_{r}=\sum_{n \geq 1}\left(\begin{array}{c}
r+n \\
r
\end{array}\right)\left[I_{r} \otimes\left(\otimes^{n} \psi\right)\right]\left(S_{r+n} x_{r+n}\right)
$$

for all $r \geq 0$. By $S_{k}$ we mean the symmetrizing operator on $\hat{E}_{k}$ for $k \geq 0$. The properties of $S_{k}$ will be discussed in the next section.

The above definition of $x(\psi)$ yields the estimate (for $u \in \lambda^{\times}, s \geq 0$ )

$$
\sum_{0 \leq r \leq N}\left|u_{r}\right| p_{s, \varepsilon}^{(r)}\left[x(\psi)_{r}\right] \leq \sum_{m \geq 1}\left[1+q_{t}(\psi)\right]^{m}(|u| * \delta)_{m} p_{s+t}^{(m)}\left(x_{m}\right)
$$

Taking the limit $N \rightarrow \infty$ proves that $x(\psi)$ is an element of $T(E, \lambda)$.

Our next step is to write the action of $\otimes^{n}(\psi+\xi)$ in terms of $\otimes^{n} \psi$ and $\otimes^{n} \xi$ and estimate the remainder. For $n \geq 2$ we observe that

$$
\begin{aligned}
& {\left[\otimes^{n}(\psi+\xi)\right]\left(S_{n} x_{n}\right)=\left(\otimes^{n} \psi\right)\left(S_{n} x_{n}\right)+\left(\otimes^{n} \xi\right)\left(S_{n} x_{n}\right)} \\
& \quad+\sum_{1 \leq r \leq n-1}\left(\frac{n}{r}\right)\left[\left(\otimes^{r} \xi\right) \otimes\left(\otimes^{n-r} \psi\right)\right]\left(S_{n} x_{n}\right)
\end{aligned}
$$

For $n=1$ the cross terms vanish, and for $n=0$ only $\psi$ appears. Hence for all $N \geq 2$ we may write

$$
\begin{aligned}
& \sum_{0 \leq n \leq N}\left[\otimes^{n}(\psi+\xi)\right]\left(S_{n} x_{n}\right) \\
& \quad=\sum_{0 \leq n \leq N}\left(\Theta^{n} \psi\right)\left(S_{n} x_{n}\right)+\sum_{1 \leq n \leq N}\left(\Theta^{n} \xi\right)\left(S_{n} x_{n}\right)+\Theta_{N} .
\end{aligned}
$$

The remainder term is

$$
\Theta_{N}=\sum_{1 \leq r \leq N-1}\left(\otimes^{r} \xi\right) \sum_{1 \leq n \leq N-r}\left(\frac{r+n}{r}\right)\left[I_{r} \otimes\left(\otimes^{n} \psi\right)\right]\left(S_{r+n} x_{r+n}\right)
$$

Then

$$
\begin{aligned}
& \Theta_{N}-m_{x(\psi)}(\xi)+m_{x(\psi)}(0)= \\
& \quad-\sum_{1 \leq r \leq N-1}\left(\otimes^{r} \psi\right) \sum_{n \geq N+1-r}\left(\begin{array}{c}
r+n \\
r
\end{array}\right)\left[I_{r} \otimes\left(\otimes^{n} \psi\right)\right]\left(S_{r+n} x_{r+n}\right) \\
& \quad-\sum_{r \geq n}\left(\otimes^{r} \xi\right)\left[x(\psi)_{r}\right] .
\end{aligned}
$$

The second term is the "tail" of the convergent series $\sum_{r \geq 0}\left(\otimes^{r} \xi\right)\left[x(\psi)_{r}\right]$ and so converges to zero as $N \rightarrow \infty$. 
We may choose $w$ in such a way that $\xi \in F_{w}\left[q_{w}\right]$. With this choice, the first term is bounded by

$$
\sum_{m \geq N+1}\left[q_{t}(\psi)+q_{w}(\xi)\right]^{m} p_{t+w}^{(m)}\left(x_{m}\right),
$$

and so converges to zero as $N \rightarrow \infty$. The result has now been shown.

Corollary 3.14. If $\lambda$ is of type $(M)$, the functions $m_{x}: E_{h}^{\prime} \rightarrow C$ are strongly continuous for all $x \in T(E, \lambda)$.

We are now ready to start our consideration of the symmetric algebra corresponding to $T(E, \lambda)$. The next section starts with a study of the symmetrizing operator.

\section{§4. The Symmetric Tensor Algebra}

The permutation group on $n$ letters, $G_{n}$, acts on $\otimes^{n} E$ by linear extension from

$$
\left[U_{g}^{(n)}\right]^{-1}\left(x_{1} \otimes \cdots \otimes x_{n}\right)=x_{g(1)} \otimes \cdots \otimes x_{g(n)} .
$$

As $U_{g}^{(n)}$ is isometric with respect to the sesquilinear form associated with $p_{r}^{(n)}$, all $r \geq 0$, it extends continuously to an operator on $\hat{E}_{n}$ written $\hat{U}_{g}^{(n)}$. The symmetrizer on $\hat{E}_{n}$ is defined to be the operator

$$
S_{n}=(n !)^{-1} \sum_{g \in G_{n}} \hat{U}_{g}^{(n)} \quad(n \geq 1) .
$$

Setting $S_{0}$ on $\hat{E}_{0}$ to be the identity map, the symmetrizer on $T(E, \lambda)$ is the operator given by

$$
S\left(x_{n}\right)=\left(S_{n} x_{n}\right)
$$

The following properties of $S$ are easily verified.

Proposition 4.1. For all $u \in \lambda^{\times}, r \geq 0, x \in T(E, \lambda)$,

$$
p_{u, r}(S x) \leq p_{u, r}(x),
$$

and so $S$ is continuous. It is $a^{*}$-projection:

$$
\begin{gathered}
S^{2}=S, \\
(S x)^{*}=S\left(x^{*}\right),
\end{gathered}
$$

and is related to the algebra product through the equations

$$
S(x y)=S(y x)=S[S(x) S(y)]=S[x S(y)]=S[S(x) y],
$$


all $x, y \in T(E, \lambda)$.

The kernel of $S, N(S)$, is a closed *-ideal, so

$$
T_{S}(E, \lambda)=T(E, \lambda) / N(S)
$$

is a locally convex Hausdorff space with the quotient topology $t_{s}\left(E, \lambda^{x}\right)$, determined by the quotient seminorms

$$
\tilde{p}_{u, r}(\tilde{x})=\inf \left\{p_{u, r}(x+y): y \in N(S)\right\},
$$

$u \in \lambda^{\times}, r \geq 0$. We have introduced the notation $\tilde{x}=x+N(S)$ for the coset equivalence classes.

The quotient seminorms satisfy the invariance equation

$$
\tilde{p}_{u, r}(\tilde{x})=p_{u, r}(S x) \text {. }
$$

If we write

$$
i: \tilde{x} \mapsto S x,
$$

then $i$ is a topological isomorphism of $T_{S}(E, \lambda)\left[t_{S}\left(E, \lambda^{\times}\right)\right]$onto the range of $S$. As $S$ is continuous, the range, $R(S)$, is a $t\left(E, \lambda^{\times}\right)$-closed subspace of $T(E, \lambda)$. The following is now obvious.

Proposition 4.2. $T_{S}(E, \lambda)\left[t_{s}\left(E, \lambda^{\times}\right)\right]$is nuclear; it is Fréchet if $T(E, \lambda)$ $\left[t\left(E, \lambda^{\times}\right)\right]$is. It can be given the structure of an abelian topological $*_{\text {-algebra with }}$ jointly continuous product by setting

$$
\begin{aligned}
\tilde{x} \tilde{y} & =(x y)^{\sim} \\
(\tilde{x})^{*} & =\left(x^{*}\right)^{\sim} .
\end{aligned}
$$

If $T(E, \lambda)$ is locally multiplicatively convex, then so is $T_{s}(E, \lambda)$.

Equipped with these operations, we shall refer to $T_{S}(E, \lambda)$ as the symmetric tensor subalgebra of $T(E, \lambda)$.

The following two combinatoric lemmas prove useful in a number of contexts. By $S_{n}^{\prime}, S^{\prime}$ we mean the transposes of $S_{n}, S$ respectively.

Lemma 4.3. If $x_{n} \in \hat{E}_{n}$ is such that $\left(\otimes^{n} \psi\right)\left(x_{n}\right)=0$ for all $\psi \in E_{h}^{\prime}$, then $S_{n} x_{n}=0$.

Proof. Let $\Delta_{n}$ be the collection of all maps

$$
\delta:\{1,2, \cdots, n\} \rightarrow\{-1,+1\} .
$$

For any $n$-tuple $\Psi=\left(\Psi_{1}, \Psi_{2}, \cdots, \Psi_{n}\right)$ of elements of $E_{h}^{\prime}$ and any $\delta \in \Delta$ we define 


$$
\Psi_{\delta}=\sum_{1 \leq j \leq n} \delta_{j} \Psi_{j},
$$

so $\Psi_{\delta} \in E_{h}^{\prime}$. We abbreviate $\Psi_{\delta}^{n}=\otimes^{n} \Psi_{\delta}$.

A multinomial expansion yields the expression

$$
\Psi_{\delta}^{n}=\sum n ! S_{n}^{\prime}\left[\bigotimes_{1 \leq k \leq n}\left(\otimes^{i(k)} \Psi_{k}\right)\right]_{1 \leq k \leq n} \frac{1}{i(k) !} \delta_{k}^{i(k)},
$$

where the sum is over all $i(k) \geq 0$ constrained by $i(1)+\cdots+i(n)=n$.

This equation may be inverted, yielding

$$
S_{n}^{\prime}\left(\Psi_{1} \otimes \cdots \otimes \Psi_{n}\right)=\left(2^{n} n !\right)^{-1} \Sigma_{\Delta_{n}} \delta_{1} \cdots \delta_{n} \Psi_{\delta}^{n} .
$$

Under the hypothesis, $\Psi_{\delta}^{n}\left(x_{n}\right)=0$ for all $\delta \in \Delta_{n}$, and from the above equation, we find that

$$
\left(\psi_{1} \otimes \cdots \otimes \psi_{n}\right)\left(S_{n} x_{n}\right)=0
$$

for all $\psi_{j}$, and so $S_{n} x_{n}=0$.

Lemma 4.4. Let $x_{n} \in S_{n}\left(\hat{E}_{n}\right)$ and $y_{m} \in S_{m}\left(\hat{E}_{m}\right)$ be such that $S_{n+m}\left(x_{n} \otimes y_{m}\right)=0$. Then if $x_{n} \neq 0, y_{m}=0$.

Proof. Choose $\psi_{1} \in E_{h}^{\prime}$ such that $\left(\otimes^{n} \psi_{1}\right)\left(x_{n}\right) \neq 0$. For any nonzero $\psi_{2} \in$ $E_{h}^{\prime}$ there exists $r>0$ such that $\left(\otimes^{n} \psi_{w}\right)\left(x_{n}\right) \neq 0$ for all $w \in \boldsymbol{R}$ with $|w|<r$, where we define $\psi_{w}=\psi_{1}+w \psi_{2}$. As $\otimes^{n+m} \psi_{w}$ is symmetric, it follows that $|w|<r$ implies $\left(\otimes^{m} \psi_{w}\right)\left(y_{m}\right)=0$. Considering this as a function of $w$ immediately yields $\left(\otimes^{m} \psi_{2}\right)\left(y_{m}\right)=0$. As $\psi_{2}$ was arbitrary, $y_{m}=0$.

Our first application of these results concerns the algebraic properties of the symmetric subalgebra.

Proposition 4.5. The algebra $T_{S}(E, \lambda)$ has no zero divisors, whence its only idempotents are 0 and $\tilde{e}$. Both $T(E, \lambda)$ and $T_{s}(E, \lambda)$ may have non-scalar units.

Proof. Consider $\tilde{x}, \tilde{y} \in T_{S}(E, \lambda)$ with $\tilde{x} \tilde{y}=0$. If suffices to consider $x, y \in$ $S[T(E, \lambda)]$ with $S(x y)=0$. Suppose $\tilde{x} \neq 0$, and let $n$ be the index of the first nonzero component of $x$.

For any $m$,

$$
0=S_{n+m}\left[\sum_{0 \leq j \leq m} x_{n+j} \otimes y_{m-j}\right],
$$

so $m=0$ yieds $y_{0}=0$. Proceeding inductively, assume $y_{0}, \cdots, y_{p-1}=0$. With $p=m$ in the above, $S_{n+p}\left(x_{n} \otimes y_{p}\right)=0$ and we know that this implies $y_{p}=0$. Hence $\tilde{y}=0$. 
For $E=C$ we have $T(C, \lambda)=\lambda$. Let $\lambda=h$, and consider the element $(1 / n !$ : $n \geq 0$ ). It is easy to verify that this is symmetric and invertible, with inverse $\left[(-1)^{n} / n !: n \geq 0\right]$.

The order properies of $T_{S}(E, \lambda)$ will be defined through the positive cone

$$
K_{S}(E, \lambda)=\left\{\sum_{j<\infty} \tilde{a}(j)^{*} \tilde{a}(j): a(j) \in T(E, \lambda)\right\} .
$$

Proposition 4.6. Under the isomorphism $i: \tilde{x} \rightarrow S x$ of equation (4.7), the positive cone may be identified with

$$
K(E, \lambda) / N(S) \cong K_{S}(E, \lambda) .
$$

The cone $K_{S}(E, \lambda)$ is generating, strict-b, and proper.

Proof. Equation (4.11) is obvious. $K_{S}(E, \lambda)$ is a generating strict-b cone because $T_{S}(E, \lambda)$ is abelian and has a jointly continuous product.

To see that it is proper, consider $\tilde{a} \in K_{S}$ with $\tilde{a} \neq 0$. There is a representation $i(\tilde{a})=S\left[\sum_{j<\infty} a(j)^{*} a(j)\right]$ with $a(j) \neq 0$ and $a(j) \in S[T(E, \lambda)]$ for every $j$. Let $L_{j}$ be the index of the first nonvanishing component of $a(j)$, and set $L=$ $\min _{j}\left(L_{j}\right)$. Then $i(\tilde{a})_{n}=0$ for $0 \leq n \leq 2 L-1$, and $i(\tilde{a})_{2 L}=S_{2 L}\left[\sum_{j<\infty} a(j)_{L}^{*} \otimes a(j)_{L}\right]$.

If $i(\tilde{a})_{2 L}$ were zero, Lemma (4.3) would imply $a(j)_{L}=0$ for all $j$, which contradicts the assumption. Thus $2 L$ is the index of the first nonvanishing component of $i(\tilde{a})$.

Suppose $\tilde{a} \in K_{S}(E, \lambda) \cap-K_{S}(E, \lambda)$, with $\tilde{a} \neq 0$. The lowest index for both $i(\tilde{a})$ and $i(-\tilde{a})$ is $2 L$ by the above reasoning, so that

$$
\left(\otimes^{2 L} \psi\right)\left[i(\tilde{a})_{2 L}\right]=\Sigma_{j}\left|\left(\otimes^{L} \psi\right)\left(a(j)_{L}\right)\right|^{2}=0
$$

for all $\psi \in E_{h}^{\prime}$, and so $a(j)_{L}=0$ for all $j, a$ contradiction.

Another application is the proof that $\hat{t}\left(E, \lambda^{\times}\right)$restricted to $T_{s}(E, \lambda)$ is Hausdorff.

Lemma 4.7. If $x \in S[T(E, \lambda)]$ is such that $M_{\psi}(x)=0$ for all $\psi \in E_{h}^{\prime}$, then $x=0$.

Proof. Considering $0=M_{t \psi}(x)$ as a function of $t \in \boldsymbol{R}$ yields $0=\otimes^{n} \psi\left(x_{n}\right)$ for all $\psi$ and all $n$, and so $x=0$.

Propostion 4.8. Write $\hat{t}_{s}\left(E, \lambda^{\times}\right)$for the topology $\hat{t}\left(E, \lambda^{\times}\right)$restricted to $T_{S}(E, \lambda)$. Then $\hat{t}_{S}\left(E, \lambda^{\times}\right)$is Hausdorff.

Proof. Let $x \in S[T(E, \lambda)]$ satisfy $\hat{\pi}_{u, r}(x)=0$ for all $u \in \lambda^{\times}, r \geq 0$. Then 
$\pi_{u, r}\left(m_{x}\right)=0$ for all $u, r$, so $m_{x}=0$. Thus $M_{\psi}(x)=0$ for all $\psi$, and Lemma (4.8) yields $x=0$.

We shall see later that $K_{S}(E, \lambda)$ is not necessarily normal. In all cases, however, its closure is proper.

Theorem 4.9. The $t_{S}\left(E, \lambda^{\times}\right)$-closed cone ${\overline{K_{S}(E, \lambda)}}^{\lambda}$ is proper.

Proof. Given $\tilde{a} \in K_{S}(E, \lambda)$ there is a $b \in K(E, \lambda)$ such that $i(\tilde{a})=S(b)$. Then $M_{\psi}[i(\tilde{a})]=M_{\psi}(b)$ is non-negative for all $\psi \in E_{h}^{\prime}$. Consider $\tilde{a} \in \overline{K_{S}(E)} \lambda$. As $M_{\psi}$ is continuous, $M_{\psi}[i(\tilde{a})] \geq 0$ for all $\psi$. If also $-\tilde{a} \in{\overline{K_{S}(E, \lambda)}}^{\lambda}$, then $M_{\psi}[i(\tilde{a})]$ $=0$ for all $\psi$, which implies that $\tilde{a}=0$.

\section{§5. Normality and Decomposability for $T_{S}(E, \lambda)$}

In this section we combine the integral decomposition theory for $T(E, \lambda)$ with the normality condition for $K(\lambda)$ in the quotient algebra $T_{S}(E, \lambda)$. As expected, everything dovetails smoothly. For the remainder of this section we assume that the cone $K(\lambda)$ is normal.

Lemma 5.1. If $\psi \in E_{h}^{\prime}$ is such that for some $u \in \Lambda^{\times}$and $r \geq 0$ the sequence $\left(q_{r}(\psi)^{n} / u_{n}\right)$ is bounded, then the function $F_{u, r}$ of equation (3.4) can be written as

$$
F_{u, r}(\psi)=\sup \left\{q_{r}(\psi)^{n} / u_{n}: n \geq 0\right\} .
$$

Proof. Now for all $x_{n} \in \hat{E}_{n}$ and $n \geq 0$

$$
\left|\left(\otimes^{n} \psi\right)\left(x_{n}\right)\right| \leq q_{r}(\psi)^{n} p_{r, \varepsilon}^{(n)}\left(x_{n}\right),
$$

and so

$$
\begin{aligned}
\left|M_{\psi}(x)\right| & \leq \sum_{n \geq 0} q_{r}(\psi)^{n} p_{r, \mathrm{\varepsilon}}^{(n)}\left(x_{n}\right) \\
& \leq p_{u, r, \mathrm{\varepsilon}}(x) \sup \left\{q_{r}(\psi)^{n} / u_{n}: n \geq 0\right\}
\end{aligned}
$$

for all $x$. Thus

$$
F_{u, r}(\psi) \leq \sup \left\{q_{r}(\psi)^{n} / u_{n}: n \geq 0\right\} .
$$

This shows that $F_{u, r}(\psi)$ is finite, and so for all $x \in T[E, \lambda]$

$$
\left|M_{\psi}(x)\right| \leq F_{u, r}(\psi) p_{u, r, \varepsilon}(x) .
$$

It follows from this inequality that

$$
\left|\left(\otimes^{n} \psi\right)\left(x_{n}\right)\right| \leq F_{u, r}(\psi) u_{n} p_{r, \varepsilon}^{(n)}\left(x_{n}\right)
$$

on $\hat{E}_{n}$, so that 


$$
q_{r}(\psi)^{n} \leq u_{n} F_{u, r}(\psi)
$$

for all $n$. The equality (5.1) is immediate.

Corollary 5.2. If $\psi \in E_{h}^{\prime}$ is on the unit sphere of $F_{r}\left[q_{r}\right]$, then $F_{\delta, r}(\psi)=1$.

As noted, we assume $\lambda$ to be such that $\tau\left(\lambda^{x}\right)=\hat{\tau}\left(\lambda^{x}\right)$. We want to similarly relate $t_{S}\left(E, \lambda^{\times}\right)$and $\hat{t}_{s}\left(E, \lambda^{\times}\right)$. For this we need two lemmas.

Lemma 5.3. For all $u \in \lambda^{\times}, r \geq 0$ the function

$$
x \mapsto \sum_{n \geq 0}\left|u_{n}\right| \hat{\pi}_{\delta, r}\left(\tilde{x}_{n}\right)
$$

is a $\hat{\imath}\left(E, \lambda^{\times}\right)$-continuous seminorm.

Proof. Given $u$, we choose $v \in \Lambda^{\times}$to satisfy

$$
\sum_{n \geq 0} 2^{n+1}\left|u_{n}\right|\left|a_{n}\right| \leq\|a\|_{v}
$$

for all $a \in \phi, c f$ equation (2.9). Given $N$ there exists a $0 \leq J \leq N$ for which

$$
\begin{aligned}
\sum_{n \leq N}\left|u_{n}\right| \hat{\pi}_{\delta, r}\left(\tilde{x}_{n}\right) & \leq 2^{J+1}\left|u_{J}\right| \hat{\pi}_{\delta, r}\left(\tilde{x}_{J}\right) \\
& \leq \sup _{\psi}\left\{\sum_{n \leq N} 2^{n+1}\left|u_{n} M_{\psi}\left(\tilde{x}_{n}\right)\right| / F_{\delta, r}(\psi)\right\} \\
& \leq \sup _{\psi} \sup \left\{M_{t \psi}\left|\left(Q^{N} x\right)\right| / F_{v}(t) F_{\delta, r}(\psi): t \in \mathbb{R}\right\}
\end{aligned}
$$

where $Q^{N}$ is the projection $Q^{N} y=\left(y_{0}, \cdots, y_{N}, 0, \cdots\right)$ and $F_{v}$ is defined in equation (2.7).

If $y$ is such that $p_{v, r, \varepsilon}(y) \leq 1$, then

$$
\left|M_{t \psi}(y)\right| \leq F_{v}(t) F_{\delta, r}(\psi) .
$$

Hence

$$
F_{v}(t) F_{\delta, r}(\psi) \geq F_{v, r}(t \psi)
$$

for all $\psi \in E_{h}^{\prime}$, and $t \in \mathbb{R}$. The double supremum, then, is bounded by $\hat{\pi}_{v, r}\left(Q^{N} x\right)$. But $\hat{\pi}_{v, r}$ is $t\left(E, \lambda^{\times}\right)$-continuous, and this enables us to take the limit $N \rightarrow \infty$, yielding

$$
\sum_{n \geq 0}\left|u_{n}\right| \hat{\pi}_{\delta, r}\left(\tilde{x}_{n}\right) \leq \hat{\pi}_{v, r}(x)
$$

finishing the proof.

Lemma 5.4. For all $v \in \Lambda^{\times}, r \geq 0, p_{r, \mathrm{\varepsilon}}^{(n)}$ satisfies the bound

$$
p_{r, \varepsilon}^{(n)}\left(S_{n} x_{n}\right) \leq(2 e)^{n} \hat{\pi}_{\delta, r}\left[\left(S_{n} x_{n}\right)^{\sim}\right]
$$

for all $x_{n} \in \hat{E}_{n}$. 
Proof. We decompose $E^{\prime}=E_{h}^{\prime}+i E_{h}^{\prime}$ in the obvious fashion. This enables us to consider hermitian functionals only, without loss of generality. Then

$$
p_{r, \mathrm{\varepsilon}}^{(n)}\left(S_{n} x_{n}\right) \leq 2^{n} \sup \left\{\left|\left(\psi_{1} \otimes \cdots \otimes \psi_{n}\right)\left(S_{n} x_{n}\right)\right|: \psi_{j} \in E_{h}^{\prime}, q_{r}\left(\psi_{j}\right) \leq 1\right\} .
$$

Let $\psi_{1}, \cdots, \psi_{n} \in E_{h}^{\prime}$ be arbitrary. Using the technique in the proof of Lemma (4.3) we can show the existence of $a \delta \in \Delta_{n}$ such that

$$
\left|\left(\psi_{1} \otimes \cdots \otimes \psi_{n}\right)\left(S_{n} x_{n}\right)\right| \leq(n !)^{-1}\left|M_{\Psi_{\delta}}\left[\left(S_{n} x_{n}\right)^{\sim}\right]\right| .
$$

As

$$
q_{r}\left(\Psi_{\delta}\right) \leq \Sigma_{j} q_{r}\left(\psi_{j}\right) \leq n
$$

we get that

$$
\begin{aligned}
\left|\left(\psi_{1} \otimes \cdots \otimes \psi_{n}\right)\left(S_{n} x_{n}\right)\right| & \leq\left(n^{n} / n !\right) M_{\Psi_{\delta}}\left(S_{n} x_{n}\right) / q_{r}\left(\Psi_{\delta}\right)^{n} \\
& \leq e^{n} \sup _{\Psi}\left\{\left|M_{\Psi / q_{r}(\Psi)}\left[\left(S_{n} x_{n}\right)^{\sim}\right]\right|: \Psi \in E_{h}^{\prime}\right\} \\
& \leq e^{n} \sup _{\Psi}\left\{\left|M_{\Psi}\left[\left(S_{n} x_{n}\right)^{\sim}\right]\right| / F_{\delta, r}(\Psi) \mid: \Psi \in E_{h}^{\prime}\right\}
\end{aligned}
$$

Substituting this into the first inequality of this proof yields the desired bound.

Now we can prove the equivalence of the topologies.

Theorem 5.5. The topologies $t_{s}\left(E, \lambda^{\times}\right)$and $\hat{t}_{s}\left(E, \lambda^{\times}\right)$are equivalent.

Proof, For any $r \geq 0$ choose $s \geq 0$ and $K>0$ to satisfy $p_{r}^{(n)} \leq K p_{s, \varepsilon^{\prime}}^{(n)}$ Let $u \in \lambda^{\times}$be arbitrary. Set $v_{n}=(2 e K)^{n} u_{n}$ and choose $w \in \Lambda^{\times}$so as to satisfy Lemma 5.3 with respect to $v$. With this notation we find that

$$
\begin{aligned}
p_{u, r}(S y) & \leq \sum_{n \geq 0} K^{n}\left|u_{n}\right| p_{s, \mathrm{e}}^{(n)}\left(S_{n} y_{n}\right) \\
& \leq \sum_{n \geq 0}\left|v_{n}\right| \hat{\pi}_{\delta, s}\left[\left(S_{n} y_{n}\right)^{\sim}\right] \\
& \leq \hat{\pi}_{w, s}(S y) .
\end{aligned}
$$

Corollary 5.6. If $K(\lambda)$ is $\tau\left(\lambda^{\times}\right)$-normal in $\lambda$, then $K_{S}(E, \lambda)$ is $t_{S}\left(E, \lambda^{\times}\right)$-normal in $T_{s}(E, \lambda)$.

Proof. We know that $S[K(E, \lambda)]$ is $\hat{t}\left(E, \lambda^{\times}\right)$-normal. From Theorem 5.5 it is $t(E, \lambda)$-normal. As the isomorphism $\tilde{x} \mapsto S x$ preserves positivity, the result follows.

We can go to the symmetric quotient of the integral decomposition as follows.

Corollary 5.7. If $R$ is a $t_{S}\left(E, \lambda^{\times}\right)$-continuous linear functional on $T_{S}(E, \lambda)$, 
we can find a finite complete complex Borel measure $\nu_{R}$ on $E_{h}^{\prime}$ such that

(i) Every weakly continuous function $E_{h}^{\prime} \rightarrow C$ is $\nu_{R^{-}}$-measurable.

(ii) Let us write $n_{S_{x}}=n_{\tilde{x}}$ for all $x$. Then $n_{\tilde{x}} \in L^{1}\left(E_{h}^{\prime}, d \nu_{R}\right)$ and

$$
R(\tilde{x})=\int_{E^{\prime}} n_{\tilde{x}} d \nu_{R} \quad\left(\tilde{x} \in T_{S}(E, \lambda)\right) .
$$

Proof. $R$ induces a $t\left(E, \lambda^{\times}\right)$-continuous linear functional $R_{1}$ on $S[T(E, \lambda)]$ defined by $R=R_{1} \circ i . \quad R_{1}$ is $\hat{t}\left(E, \lambda^{\times}\right)$-continuous and so extends continuously to $T(E, \lambda)$. The result now follows from Proposition 3.7.

\section{References}

[1] Alcantara, J. and Dubin, D.A., I*-algebras and their applications. Publ. RIMS Kyoto Univ., 17 (1981), 179-199.

[2] Borchers, H.J., On the structure of the algebra of field operators. Il Nuovo Cimento, 24 (1962), 214-236.

[3] Borchers, H.J. and Yngvason, J., Necessary and Sufficient Conditions for Integral Representations of Wightman Functionals at Schwinger Points. Commun. Math. Phys., 47 (1976), 197-213.

[4] Dubin, D.A. and Hennings, M.A.: Regular Tensor Algebras. Publ. RIMS Kyoto Univ., 25 (1989), 971-999.

[5] Köthe, G.: Topological Vector Spaces, I. Springer-Verlag, Berlin (1969).

[6] Uhlmann, A., Über die Definitionen des quantenfelder nach Wightman und Haag. Wiss. Z. KMU (Leipzig) 11 (1962), 213-217. 